\title{
Report on the first international workshop on the CCN family of genes
}

The first international workshop on the CCN family of genes brought together 75 participants coming from essentially all laboratories working in this expanding field. The meeting was a great success, with exceptionally good quality scientific exchange, and many fruitful personal contacts being made.

As can be seen in this meeting summary, major progress has been made in the fields of development and pathobiology. New aspects concerning the structural basis for some functions of the $\mathrm{CCN}$ proteins and their conservation emerged and most certainly constitute exciting avenues for future research in the CCN field.

\section{Role of the CCN proteins in developmental processes}

Several reports have confirmed the importance of CCN proteins in the chondrogenesis and ossification processes.

Early studies by L Lau and collaborators had established that CYR61 was involved in chondrogenesis. The results that were presented at this meeting indicated that cyr61 is expressed in trophoblasts and spongioblasts and established a role for CYR61 in the formation of the chorion and its vascularisation, as shown by the effects of the recombinant CYR61 protein on cell proliferation, migration, and attraction of endothelial cells (as shown in in vitro cultures or grafts). Cyr61 knockout of mice resulted in embryonic lethality as a result of vascular defects in both embryonic and extraembryonic tissues.

A major role for CYR61 appears to be in bone signalling. It is produced by mesenchymal cells and osteoclasts from fracture callus, in matured growth plate chondrocytes, in proliferating osteoblast cell lines, and in primary cultures. Moreover, expression of cyr61 is stimulated in osteoblasts by vitamin D3 and growth factors, all of which are important in bone metabolism (presented by N Scuhtze).

Results presented by $M$ Takigawa established that connective tissue growth factor (CTGF; highly expressed in chondrocytes, and designated Hcs 28 by the authors) is involved in skeletogenesis not only during prenatal development but also under traumatic conditions and in bone fracture repair. The effects of recombinant CTGF and corresponding antibodies on proliferation and differentiation of chondrocytes (proteoglycans and collagen synthesis), osteoblasts (mineralisation), and endothelial cells (proliferation, migration, and angiogenesis) were assessed using in vitro cultures. A paracrine effect of CTGF on three cell lines indicated involvement in endochondral ossification. The role of CTGF in fracture repair suggests future clinical applications.

In a similar manner to CYR61, CTGF also figures prominently in vascularisation (endothelial cells and myocardium) and cartilage development (presented by $\mathrm{K}$ Lyons). CTGF is strongly expressed in skeletal growth plates, in particular, in the context of chondrocyte hypertrophy, a necessary maturation step for endochondral ossification. Overexpression of ctgf after transfection interfered with the proliferation of stromal cells in the bone marrow, articular chondrogenesis, and cartilage hypertrophy, depending on the degree of expression and in association with transforming growth factor $\beta$ (TGF- $\beta$ ). Further confirmation was obtained from ctgf knockout studies in mice, where lack of CTGF led to skeletal defects affecting ribs, sternum, cervical vertebrae, and craniofacial elements. Because CTGF might also induce vascular ingrowth into the growth plate, it probably plays an important role in many aspects of bone growth and repair.

Expression of ctgf in newt limb regeneration with or without denervation was described in a poster presented by Moussad et al. Early ctgf expression was detected in different cell types. A role for CTGF was proposed in morphogenesis (digit indentation of stump) and hypertrophy of cartilage. Increased ctgf expression after denervation resulted in muscle atrophy. In this system, CTGF was shown to play a role in the inflammatory response, extracellular matrix remodelling, cell proliferation, endochondral ossification, and morphogenetic cell death.

CTGF-L/rCOP-1/WISP-2 also appears to be implicated in osteoblast and chondrocyte function because it is found in high amounts in osteoblasts and stimulates adhesion and mineralisation of osteoblasts and proteoglycan synthesis in chondrocytes (presented by S Kumar).

NOV also appears to be involved in chondrocyte function and differentiation because it is found in the growth plate as well as in endochondromas, where expression was generally higher in low rather than high grade lesions. In chondrocyte organ cultures, nov expression was stimulated by parathyroid hormone related protein (PTHrP), a regulator of growth plate chondroctye differentiation (presented by B Alman). In addition, nov is expressed during the first trimester in the developing musculoskeletal system including the spinal cord, skeletal and smooth muscle, and chondrocytes (presented by $\mathrm{P}$ Schofield).

At early stages of development in the chick embryo, the expression of nov was detected in dorsal and periventricular aspects of the neural tube, from 2 days onwards (presented by $\mathrm{K}$ Katsube). With this exception, nov expression was restricted to mesodermally derived structures (predominant in somites and myotomes in areas from which the hypaxial musculature originates). NOV was also found in the notochord, in the apical ridges of limb buds, and in the posterior part of branchial arches, particularly in the second arch. Cos7 cells expressing nov and grafted into the flank of the embryo induced a supernumerary limb bud-like structure. In ovo electroporation experiments showed that nov is regulated by Wnt1 (reminiscent of WISP genes being targets of Wnt regulation). This supports an involvement of NOV in ectomesenchymal interactions with a possible dorsalising effect.

As in the chick, the expression of nov is essentially detected in musculoconnective tissues in the lateral part of dermomyotomes in early mouse embryos and later on in the hypaxial muscles, ocular muscles, and blood vessel walls (presented by E Andermacher). The pax3 and nov expression patterns suggested that PAX3 and NOV are functionally connected in hypaxial myogenesis. Overexpression of nov in embryonic stem (ES) cells in vitro stimulates cell proliferation and survival and early myogenesis, but blocks terminal muscular differentiation and the formation of myotubes. Similarly, chimaeric mouse fetuses, obtained from the fusion of normal and nov ${ }^{+} \mathrm{ES}$ cells, presented with severe malformation in the craniobrachial and limb muscles. The systems involved in the negative regulation of nov at late stages of myogenesis that allow the terminal differentiation of muscle are currently being studied. 
The effects of NOV on in vitro muscle differentiation were exemplified by the capacity of full length mouse NOV protein to induce a block of differentiation of $10 \mathrm{~T} 1 / 2$ fibroblasts, as opposed to truncated NOV, which induced complete differentiation (poster presented by D Tahri). Differential expression of NOV was also observed in satellite stem cells undergoing muscle differentiation, and in muscle regeneration (poster presented by Cherel et al).

A striking example for the conservation of CCN protein functions throughout species was provided by studies performed with Xenopus laevis (presented by B Latinkic). Cloning and sequencing of cyr61 in this species had revealed a high phylogenetic conservation of this gene. In situ hybridisation allowed the detection of maternal cyr6 1 RNAs up to the cleavage stage and embryonic ones from the neurulation stage, essentially in the dorsal aspect of the embryo in mesodermal structures first (somites, notochord, branchial arch mesenchyme). Later on (from the tail bud stage) the expression was also detected in ectodermal tissues (otic vesicles and neural tube) and finally in the heart of the tadpole. A miss-expression of the cyr61 gene (complete or partial) induced by injections in the ventral regions of the embryo caused several malformations, pointing to a role for CYR61 in the dorsalisation processes of the early embryo, possibly interfering with the wnt pathway. In addition, the CYR61 domains were differentially implicated: domains 1 and 2 in the axial malformations and development of ectopic muscle and domain 4 in the blastopore closure.

$S$ Morais da Silva presented the characterisation of a new CCN-like gene and its role in an original system of "postnatal organogenesis"; the regeneration of a sectioned limb in the newt. This CCN-like gene, 70/71, was expressed in the blastema several days after the lesion and retinoic acid treatment, with a heterogenous proximodistal distribution, supporting the hypothesis for the role of this gene in the proximodistal identity of the blastema cells and possibly in epithelial-mesenchymal interactions.

Although more distantly related, both tsg (twisted gastrulation) and sog (short gastrulation) drosophila proteins share sequence homology with members of the CCN family. L Marsh reported that expression of the tsg gene can enhance the antagonistic activity of sog towards bone morphogenetic proteins (BMPs). Interestingly, tsg homologues have been cloned from zebrafish and mammalian species, and have been shown to be functionally equivalent.

\section{CCN proteins and signalling}

New results were also offered concerning the potential receptor and signalling pathways involving CCN proteins.

$\mathrm{T}$ Grzeszkiewicz presented data describing the integrin receptors that are involved in CYR61 induced activities in primary human skin fibroblasts. Integrin $\alpha 6 \beta 1$ and heparan sulphate proteoglycans were found to mediate the adhesion of fibroblasts to CYR61. CYR61 induced migration of fibroblasts was mediated by integrin $\alpha v \beta 5$, whereas integrin $\alpha v \beta 3$ is required for the enhancement of basic fibroblast growth factor (bFGF) stimulated fibroblast mitogenesis. These findings suggest that CYR61 induces different biological effects through its interaction with distinct integrin receptors.

In a similar study, presented by S Lam, integrin $\alpha_{M} \beta_{2}$ was found to be necessary for the adhesion of peripheral blood monocytes and THP-1 cells to CYR61 or CTGF. The observation that both CTGF and CYR61 proteins are found in advanced atherosclerotic lesions and wounds suggests that the interaction of monocytes with these proteins might have important implications for monocyte function during pathological conditions such as inflammation, wound healing, and atherosclerosis.

The expression of the WISP genes had been reported to be regulated by Wnt. In addition to nov being another target of Wnt regulation (see above), a novel gene, wise, bearing a weak homology to the CCN family members may play a role in the Wnt signalling pathway because immunoprecipitation studies suggest it binds to the Wnt receptor Frizzled (presented by N Itasaki ). W Kutz examined the expression pattern of WISP-3 in eukaryotic cells. Previous work has shown that loss of function mutations in WISP-3 are found in patients with the heritable skeletal disorder progressive pseudorheumatoid dysplasia, implicating a role for WISP-3 in skeletal growth and homeostasis.

A large number of reports now indicate that ctgf expression is regulated by TGF- $\beta$ in many instances. However, an increasing body of evidence suggests that this relation is not obligatory. A TGF- $\beta$ response element that is distinct from the previously identified TGF- $\beta$ response element was discussed. A Leask showed that the induction of CTGF by TGF- $\beta$ is repressed by tumour necrosis factor $\alpha$ (TNF- $\alpha$ ) through a nuclear factor $\kappa \mathrm{B}(\mathrm{NF} \kappa \mathrm{B})$ dependent pathway, and that the regulation of ctgf by TGF- $\beta$ involves SMAD binding to another distinct $\mathrm{TGF}-\beta$ responsive element.

CTGF expression in punch biopsies was strongly correlated with the presence of granulation tissue fibroblasts, peaking around seven to 10 days after injury. During the granulation phase, CTGF expression was correlated temporally and spatially with TGF- $\beta$ expression by fibroblasts, rather than TGF- $\beta$ expression by inflammatory cells during the first few days after injury (presented by $\mathrm{N}$ Hayashi). Gingival overgrowth in patients receiving phenytoin, but not nifedipine or cyclosporin, showed high CTGF expression, suggesting that different molecular mechanisms underlie the fibrotic reaction to each drug. In cultured gingival cells, TGF- $\beta$ increases collagen mRNA, lysyl oxidase mRNA values and activity, and CTGF production. However, CTGF itself only increased the amount of insoluble collagen and lysyl oxidase activity, without concomitant increases in their mRNAs, showing that CTGF complements the activities of TGF- $\beta$ but does not mediate all the profibrogenic effects of TGF- $\beta$ (presented by P Trackman).

A similar relation between CTGF and TGF- $\beta$ occurs in mesangial cells (MCs): CTGF appears to stimulate MC migration and TGF- $\beta$ induced fibronectin production, but not $\alpha$ smooth muscle actin ( $\alpha$ SMA) production or cell proliferation, which are induced by TGF- $\beta$ and platelet derived growth factor (PDGF), respectively (presented by I Bloom). Induction of CTGF in MCs and human renal fibroblasts is stimulated in a protracted manner by TGF- $\beta$ and transiently by lysophatidyic acid, the latter involving heptahelical receptors. CTGF expression by both stimuli was dependent on members of the Rho family as well as an intact cytoskeleton (presented by M Goppelt-Struebe). Rho, together with protein kinase $\mathrm{C}$ and phosphatidylinositol 3-kinase (PI-3 kinase), also appears to be involved in mediating CYR61 production in smooth muscle cells (SMCs) that have been exposed to stretch forces. Whereas ctgf and cyr61 are serum inducible immediate early genes in SMCs, the application of stress forces to cultured SMCs results in a rapid and pronounced but transient increase in cyr61 expression, but protracted downregulation of ctgf gene expression. Thus cyr61 and ctgf are differentially regulated by mechanical forces and might have distinct roles in processes such as mechanotransduction or phenotypic modulation (presented by B Chaqour). 


\section{Structural basis for the biological activities of the CCN proteins}

Structural studies concerning CCN proteins are blossoming. As stated in the introductory session by B Perbal, the multimodular structure of the CCN protein presents a remarkable biochemical challenge and identifying CCN protein partners should permit the fundamental questions regarding the biological activities of these proteins to be answered.

G Grotendorst presented data showing that the DNA synthesis promoting activity of CTGF in NRK fibroblasts is absolutely dependent upon the presence of another growth factor, epidermal growth factor (EGF). This finding is consistent with similar observations made with CYR61, thus showing that these CCN proteins are not, by themselves, mitogenic growth factors. CTGF can be cleaved by plasmin into $\mathrm{N}$-terminal and $\mathrm{C}$-terminal domains. Whereas the N-terminal domain was found to exert effects on collagen synthesis, the C-terminal domain modulates DNA synthesis.

Proteolytically processed CTGF fragments of 16$20 \mathrm{kDa}$ containing the C-terminal domain (module IV) have been observed in porcine uterine flushings by $\mathrm{D}$ Brigstock. These fragments can be produced in a cell culture system using Chinese hamster ovary $(\mathrm{CHO})$ cells transfected with an expression vector that encodes the full length protein.

L Desnoyers presented data showing that the newly identified CCN family member, WISP-1, interacts directly with decorin and biglycan. WISP-1 was found to possess a specific binding site for dermatan sulphate that is responsible for its interaction with these dermatan sulphate proteoglycans. These data suggest that decorin and biglycan may modulate WISP-1 activity in tumours where these proteins are overexpressed.

\section{Pathobiology of CCN proteins}

R Lupu reported that CYR61 expression was detected in about $30 \%$ of invasive human breast tumour biopsies. Expression of cyr61 is greatly increased in heregulin transfected MCF7 breast cancer cells, and anti-CYR61 antibodies abolish the invasiveness and migration of these cells in vitro. Moreover, CYR61 is sufficient to confer the oestrogen independence and anti-oestrogen resistance of breast cancer cells. These results suggest that CYR61 might play an important role in breast cancer progression. C Wenger observed that both cyr61 and ctgf mRNAs are moderately overexpressed in chronic pancreatitis and highly overexpressed in pancreatic cancer.

The pattern of nov expression was established in a series of different tumours. $\mathrm{H}$ Yeger described refined methods of analysing NOV expression in normal and tumoral human tissues by means of in situ hybridisation and immunocytochemistry. It was found that a brief treatment of tissue sections in the microwave resulted in enhanced signals for the detection of NOV in both cases. In some cases, the degree of NOV expression was found to correlate with increased tumorigenicity and proliferative capacity (as presented by Manara, and reported in posters by Cadot et al, Glukhova et al), whereas in other cases, such as Wilms's tumours and neuroblastomas, the expression of NOV correlated with tumour differentiation (as presented by B Perbal and by $\mathrm{H}$ Yeger). The expression of NOV in adrenocortical adenomas and carcinomas was variable, without a clearcut association between levels of expression and tumour stages (reported in a poster by Kyurkchiev et al).

$\mathrm{Y}$ Ito reported that CTGF expression is strongly upregulated in human proliferative and fibrogenic renal disease. In culture, induction of CTGF in rat mesangial cells and glomerular visceral epithelial cells by TGF- $\beta 1$,
TGF- $\beta 2$, and TGF- $\beta 3$ was observed. The role of CTGF in renal fibrosis was also reported by B Riser. Mesangial cells can be stimulated by TGF- $\beta$, a high glucose concentration, or cyclic stretch to express CTGF which, in turn, can increase the production of fibronectin and collagen type I. A 27-fold increase in CTGF expression was also observed in $\mathrm{db} / \mathrm{db}$ mice after 3.5 months of diabetes as compared with the normal $\mathrm{db} / \mathrm{m}$ control animals. In a similar vein, the urinary CTGF concentrations were measured in patients with diabetic nephropathy, and it was suggested that CTGF values may be a useful predictor of patients destined for progressive nephropathy and endstage disease.

$\mathrm{V}$ Paradis described a rat model of $\mathrm{CCl}_{4}$ induced liver fibrosis, where CTGF expression was correlated with the late stages of septal fibrosis and cirrhosis. Furthermore, in a group of 39 liver biopsies from patients with chronic hepatitis C, 21 cases showed moderate or strong CTGF expression, as did 21 of 28 liver biopsies from patients with chronic liver diseases, irrespective of aetiology.

The potential role of CTGF in systemic sclerosis, or scleroderma, was discussed by $\mathrm{K}$ Takehara. CTGF concentrations were found to increase in the sera of patients with scleroderma compared with normal controls. Subcutaneous injections of TGF- $\beta$ into mice resulted in the transient formation of granulation tissue that disappears over several days. Injection of TGF- $\beta$ and CTGF, however, resulted in persistent fibrotic tissue formation. A two step fibrosis model was proposed in which TGF- $\beta$ induces fibrosis, whereas CTGF is involved in the maintenance of fibrosis.

\section{Conclusions}

Taken together, the results presented at the first international workshop on CCN proteins have highlighted some exciting aspects of the functions of CCN genes.

If not primary players, the CCN proteins probably have a role as effectors during the embryonic and fetal development of nerves, muscle, bone marrow, and bone.

The widespread expression of the CCN genes in a diversity of cell types also suggests a complexity of molecular functions yet to be discovered. That CCN proteins act in a large variety of tissue and cell types and across a broad species range should generate increased interest in their study.

The first international workshop on the CNN family of genes was supported by grants from Ligue Nationale Contre le Cancer (Comité du Cher), Université Paris - D. Diderot, Munin Corp, Fibrogen, Genentech, Glaxo Wellcome, and Promega.

C AYER-LELIEVRE Faculté de Medecine, 87025 Limoges, France

D BRIGSTOCK Department of Surgery, Ohio State University, Colombus, Ohio 43205,

L LAU Department of Molecular Genetics, University of Illinois, Chicago, IL 60607, USA D PENNICA

Department of Molecular Oncology, Genetech, San Francisco, CA 94080

B PERBAL

Laboratoire d'Oncologie Virale et Moleculaire, UFR de Biochimie, Université Paris 7-D. Diderot, 2 Place Fussieu, 75005 Paris, France Bernard.Perbal@wanadoo.fr

H YEGER

Department of Paediatric Laboratory Medicine, Hospital for Sick Children, Toronto, Ontario M5G $1 X$ G, Canada 